\title{
Tratamento cirúrgico de dentes supranumerários na região anterior da maxila em paciente pediátrico: estudo de caso
}

\author{
Surgical treatment of supernumerary teeth in the anterior maxilla region in a pediatric \\ patient: case study
}

Tratamiento quirúrgico de dientes supranumerarios en la región anterior del maxilar en un paciente pediátrico: estudio de caso

Jorge Sá Elias Nogueira ${ }^{1 *}$, Ana Cecília Sobrinho Lima', Rebeca Feliciano Silva Rodrigues ${ }^{1}$, Pedro Aleixo Nogueira', Ricardo Roberto de Souza Fonseca², Ana Carolina Carneiro Cardoso', Isabela Braga Valente ${ }^{1}$, Silvio Augusto Fernandes de Menezes ${ }^{1}$, Suelly Maria Mendes Ribeiro ${ }^{1}$, Doris Kós Bulamarqui de Miranda'.

\section{RESUMO}

Objetivo: Relatar um caso clínico raro de tratamento cirúrgico de três dentes supranumerários na região anterior da maxila em paciente pediátrico. Detalhamento de Caso: Paciente do sexo masculino, 8 anos de idade, compareceu à urgência do Centro Universitário do Estado do Pará (CESUPA) queixando-se de anormalidade na dentição. Ao exame clínico constatou-se a irrupção dos elementos 11 e 12, um mesiodens na linha média da maxila e a ausência dos elementos 21 e 22. O exame radiográfico evidenciou três elementos supranumerários inclusos, dentre os quais, dois localizavam-se no rebordo alveolar anterior, impedindo a irrupção dos incisivos central e lateral esquerdos. O paciente foi submetido à exames sistêmicos prévios e posteriormente ao tratamento cirúrgico de exodontia dos elementos supranumerários, seguiu sob acompanhamento durante 1 ano apresentando re-erupção dos elementos 21 e 22 e subsequente encaminhamento para a Ortodontia. Considerações finais: Os exames clínicos e radiográficos periódicos são fundamentais para a obtenção do diagnóstico precoce de anomalias dentárias e correto direcionamento no planejamento cirúrgico.

Palavras-chave: Dente supranumerário, Odontopediatria, Cirurgia bucal.

\begin{abstract}
Objective: To report a rare clinical case of three supernumerary teeth in the anterior region of the maxilla in a pediatric patient. Case Details: Male patient, 8-years-old, attended to the urgency of the University Center of the State of Pará (CESUPA) complaining of abnormal dentition. The clinical examination revealed the eruption of 11 and 12 elements, a mesiodens in the midline of the maxilla and the absence of 21 and 22 elements. The radiographic examination showed three supernumerary elements included, among which, two were located in the alveolar ridge anterior, preventing the eruption of the left central and lateral incisors. The patient underwent previous systemic examinations and after the surgical treatment of extraction of the supernumerary elements, followed up for 1 year, showing re-eruption of 21 and 22 elements and subsequent referral to Orthodontics. Final considerations: Periodic clinical and radiographic examinations are essential to obtain an early diagnosis of dental anomalies and correct guidance in surgical planning.
\end{abstract}

Key words: Supernumerary teeth, Pediatric dentistry, Oral surgery.

${ }^{1}$ Centro Universitário do Estado do Pará (CESUPA). Belém - Pará. *E-mail: jorgenogueira@superig.com.br ¿2Universidade Federal do Pará (UFPA). Belém - Pará. 


\section{RESUMEN}

Objetivo: Informar un caso clínico raro de tratamiento quirúrgico de tres dientes supernumerarios en la región anterior del maxilar en un paciente pediátrico. Detalles del caso: Un paciente masculino, de 8 años de edad, asistió a la sala de emergencias en el Centro Universitario del Estado de Pará (CESUPA) informando anormalidades en sus dientes. El examen clínico reveló la erupción de los elementos 11 y 12, un mesiodens en la línea media del maxilar y la ausencia de los elementos 21 y 22. El examen radiográfico mostró tres elementos supernumerarios incluidos, entre los cuales, dos estaban ubicados en la cresta alveolar anterior, evitando la erupción de los incisivos centrales y laterales izquierdos. El paciente se sometió a exámenes sistémicos previos y después del tratamiento quirúrgico de extracción de los elementos supernumerarios, siguió durante 1 año, mostrando la erupción de los elementos 21 y 22 y la derivación posterior a la ortodoncia. Consideraciones finales: Los exámenes clínicos y radiográficos periódicos son esenciales para obtener un diagnóstico temprano de anomalías dentales y una dirección correcta en la planificación quirúrgica.

Palabras clave: Diente supernumerario, Odontología pediátrica, Cirugía oral.

\section{INTRODUÇÃO}

Os dentes supranumerários (DSN) são distúrbios dentários de desenvolvimento de número caracterizado pela presença de um ou mais elementos dentários no arco dentário quando este já tiver mais do que 20 dentes na dentição decídua e 32 na dentição permanente (DAS D e MISRA J, 2012). Apesar de bem documentada na literatura sua etiologia ainda não é totalmente conhecida, contudo estima-se que a formação de DSN podem ocorrer devido a fatores como hiperdesenvolvimento da lâmina dentária, hereditariedade, dicotomia do germe dentário e pacientes sindrômicos (RALLAN M, et al., 2013; WAINGADE M, et al., 2014).

Os DSN podem ser classificados de acordo com sua localização no arco dentário, sendo assim são conhecidos como: mesiodens (localizados linha média da maxila entre os incisivos centrais), distomolar (localizado distalmente aos terceiros molares), paramolar (localizado nas regiões vestibular ou palatina/ lingual a um molar) e suplementares (demais regiões) (WAINGADE M, et al., 2014).

Destes os mesiodens são o tipo mais prevalente e é comum que os DSN permaneçam impactados (sendo diagnósticos em exames de rotina), ou então venham a erupcionar invertidos ou em posição ectópica (BARGALE SD, et al., 2015; COSME-SILVA L, et al., 2016).

Com relação a prevalência, esta pode variar entre 0,1 e 3,8\% na população, sendo mais incidente no gênero masculino (2:1) e pode ser encontrado mais frequentemente na dentição permanente $(5: 1)$ e numericamente as manifestações podem ocorrer na maioria com a presença de até 2 DSN sendo três ou mais supranumerários menos 1\% dos casos (SARNE O, et al., 2018; GURLER G, et al., 2017).

Como supracitado os mesiodens são os DSN mais prevalentes, sendo sua taxa de aparição entre $0 \%$ a 0,19\% na dentição decídua e $0,15 \%$ a 3,8\% na dentição permanente, sendo mais incidente no gênero masculino (INDIRA M, et al., 2014). De acordo com a literatura sua prevalência é maior na dentição permanente devido ao diagnóstico tardio sendo comum sua detecção em exames de rotina (MANGALEKAR $\mathrm{SB}$, et al., 2013). Os mesiodens podem ser classificados de acordo com seu formato: igual ao dente comum com uma coroa e raiz normal ou cônico e raiz dilacerada, além de alguns casos apresentarem morfologias rudimentares semelhantes à odontomas (THOMAIDIS V, et al., 2019).

O diagnóstico dos DSN geralmente ocorre em exames de rotina nos pacientes pediátricos durante a dentição mista ou permanente, é comumente no decorrer do exame clínico que sinais e sintomas como irrupção dentária ou mau posicionamento dentário são avaliados e frequentemente exames complementares como: radiografias panorâmicas, oclusais, periapicais e tomografias computadorizada podem ser utilizadas para fechar diagnóstico. Uma vez corretamente diagnosticado um plano de tratamento adequado pode ser montado e executado, assim futuramente favorecendo o prognóstico do paciente (MADDALONE M, et al., 2018; HAUER L, et al., 2019). 
O tratamento dos DSN dependerá da localização, morfologia, presença de complicações clínicas e idade do paciente. Entre os métodos mais utilizados temos a proservação e acompanhamento do caso para justamente evitar atos cirúrgicos em pacientes pediátricos bem como uso de terapias coadjuvantes como a ortodontia para tentar a erupção ativa do DSN, já em outros caso a abordagem cirúrgica faz-se como padrão ouro de tratamento a fim de prevenir possíveis má-oclusões, tumores odontogênicos ou demais complicações (MADDALONE M, et al., 2018; HAUER L, et al., 2019; OZDEN MC, et al., 2017).

Diante disso, o objetivo deste relato é reportar um caso clínico raro de tratamento cirúrgico em um paciente do sexo masculino, de 8 anos de idade, que apresentava três DSN inclusos que impediam a irrupção dos incisivos central e lateral superiores esquerdos.

\section{DETALHAMENTO DE CASO}

Paciente do sexo masculino, 8 anos, melanoderma, normoreativa e morador da região rural do estado do Pará acompanhado por seu responsável procurou atendimento odontológico na clínica de urgência do Centro Universitário do Estado do Pará (CESUPA) com a queixa principal de ausência dos elementos 21 e 22, má posicionamento do elemento 11 e segundo a mãe "dente em formato anormal" que ainda segundo sua responsável incomodava o paciente na língua durante sua mastigação e fala.

Durante sua primeira consulta foi realizado o exame clínico intraoral constatou-se a ausência dos elementos 21 e 22, erupção do elemento 11 fora do arco dentário e levemente vestibularizado, além disso pode-se observar clinicamente a presença de um mesiodens na linha média da maxila, paulatinamente em relação ao dente 11, tumefação na região anterior da maxila sem alterações de coloração tecidual e ausência de sangramento, supuração ou dor sendo o diagnóstico sugestivo de mesiodens (Figura 1).

Durante consulta inicial percebeu-se que o paciente apresentava braquicardia e bradipneia sendo assim exames complementares foram solicitados para avaliar sua saúde sistêmica e local: A radiografia panorâmica (Figura 2A) revelou deslocamento do elemento 21 anormalmente acentuado para distal em relação à linha média e imagem radiopaca disforme multinucleada na região da linha média impactando os elementos $21 \mathrm{e}$ 22.

As radiografias periapicais foram feitas pela técnica de Clark para divergir os achados radiográficos (Figura 2B, 2C e 2D), pode-se observar 3 elementos radiopacos semelhantes a DSN no rebordo alveolar, impedindo a erupção do 21 e 22, sem quaisquer demais alterações presentes na região. Logo após avaliar dados clínicos e radiográficos o diagnóstico foi de mesiodens e como tratamento recomendou-se a remoção cirúrgica.

Figura 1 - Imagem inicial demonstrando ausência dos elementos 21 e 22 e irrupção do 11 e de 1 mesiodens.

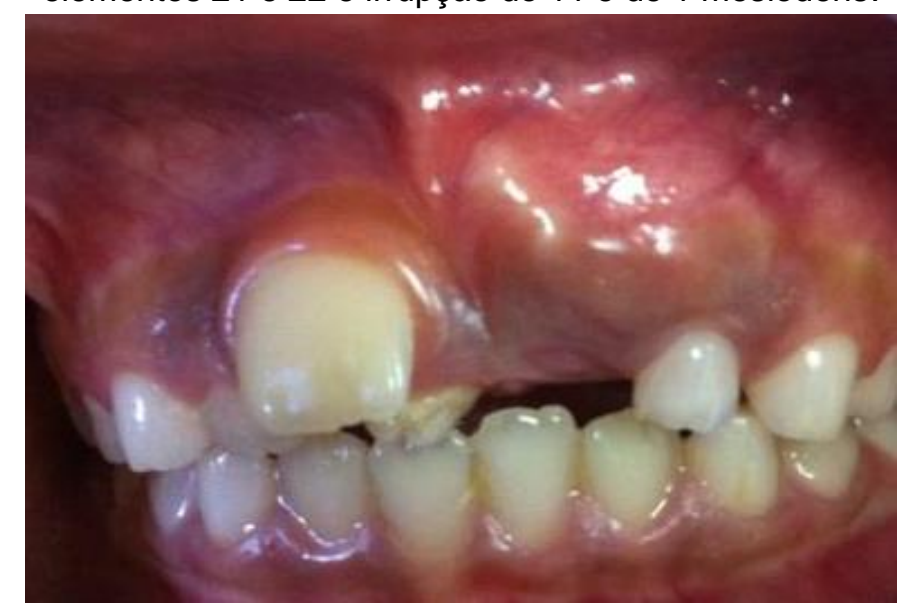

Fonte: Nogueira JSE, et al., 2020. 
Figura 2 - 2A: imagem radiopaca disforme multinucleada na região da linha média impactando elementos 21 e 22/ 2B: mesioradial/ 2C: orto-radial / 2D: disto-radial demonstrando presença de 3 DSN.

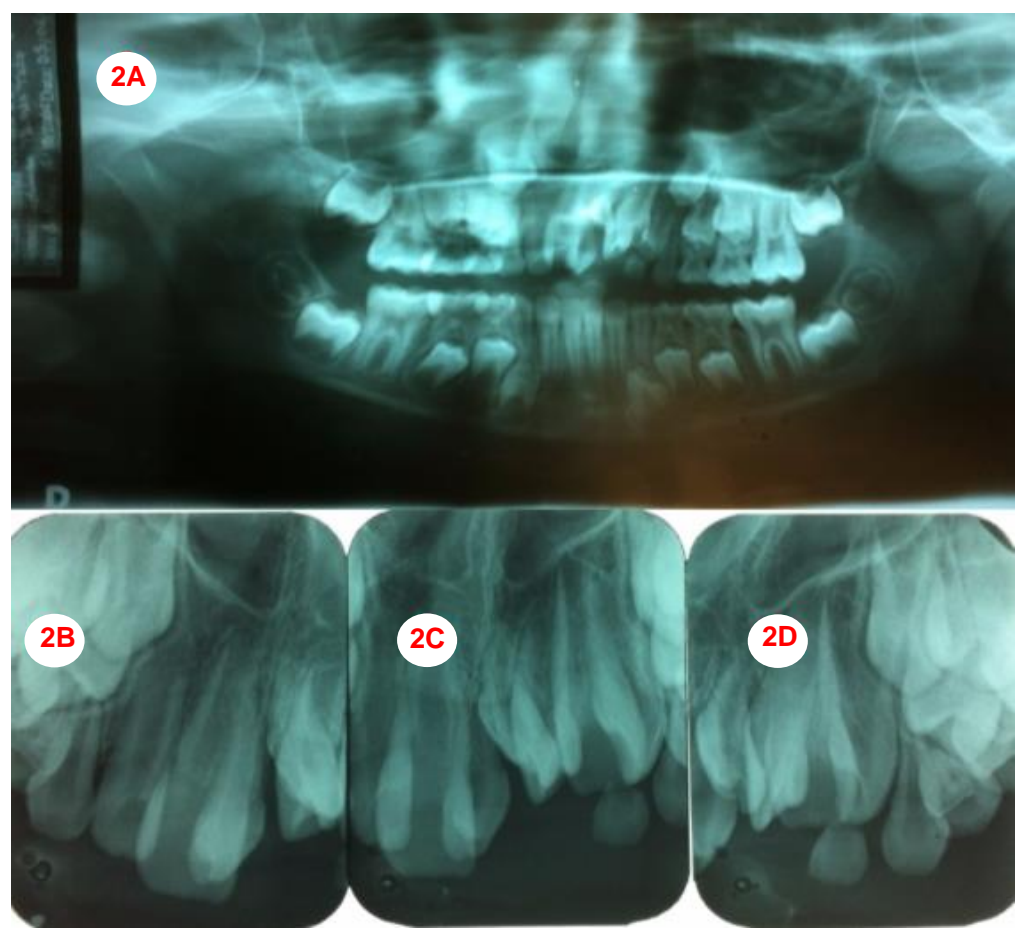

Fonte: Nogueira JSE, et al., 2020.

Os exames complementares hematológicos demonstraram ausência de quaisquer patologias sistêmicas (risco cirúrgico ASA I) que pudessem ser a causa dos sintomas apresentados na consulta inicial sendo então seu comportamento associado a tensão e nervosismo. Posteriormente realizou-se assepsia extraoral e intraoral com clorexidina $0,12 \%$ e e colocação do campo cirúrgico em seguida administrou-se anestesia local com 3 tubetes de mepivacaína 3\% com adrenalina 1:100.000 nos nervos infraorbital bilateralmente e nasopalatino. Após analgesia confirmada confeccionou-se a incisão (Figura 3) com lâmina 15c no topo de rebordo, intrasucular e com relaxante no dente 63, depois o descolamento total ocorreu com auxílio do sindesmótomo até a exposição dos DSN.

Figura 3 - Loja radiográfica evidenciando a exodontia dos 3 mesiodens.

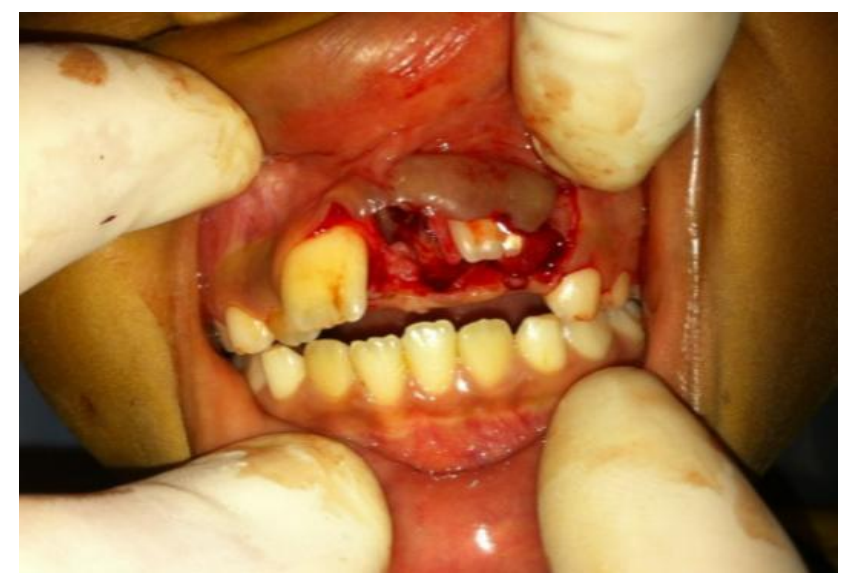

Fonte: Nogueira JSE, et al., 2020. 
Em seguida os três elementos supranumerários foram luxados com auxílio de alavancas retas e a exodontia ocorreu da forma menos traumática possível (Figura 4). Posteriormente, a região foi inspecionada e irrigada com soro fisiológico $0,9 \%$ para remover tecido e espículas ósseas e/ou tecido de granulação. 0 retalho foi readaptado e foi realizada a sutura com fio de seda 4.0. O paciente foi medicado com Ibuprofeno $600 \mathrm{mg}$ para prevenção e controle de dor e tanto o próprio paciente quanto seus responsáveis foram orientados quanto aos cuidados no pós-operatório.

No controle pós-operatório com 7 dias o paciente retornou e relatou não sentir incômodos durante sua recuperação e após remover as suturas realizou-se nova avaliação clínica e radiográfica evidenciando remoção total dos DSN e recuperação favorável (Figura 5A e 5B). Depois de 12 meses de controle observamos erupção completa dos dentes 12, 11, 21 e 22 próximo ao normal contudo pode-se notar um leve apinhamento (Figura 6A e 6B) portanto paciente foi encaminhado à ortodontia.

Figura 4 - Os três elementos supranumerários extraídos no ato cirúrgico.

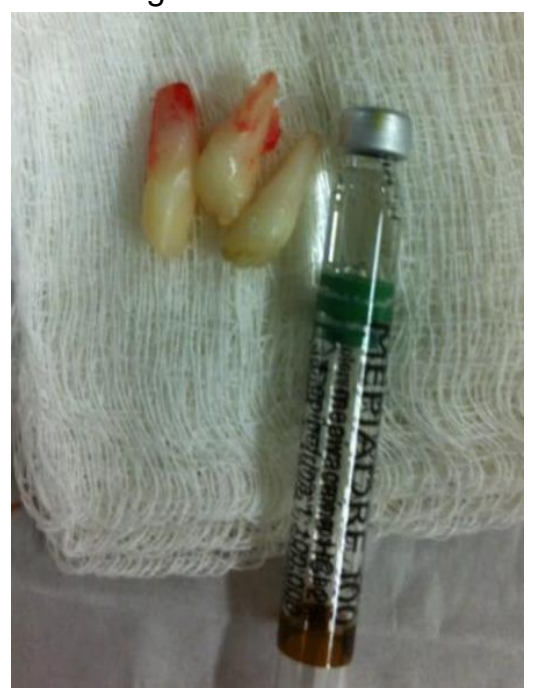

Fonte: Nogueira JSE, et al., 2020.

Figura 5A/5B - Avaliação clínica e radiográfica após 7 dias evidenciando remoção total dos DSN.

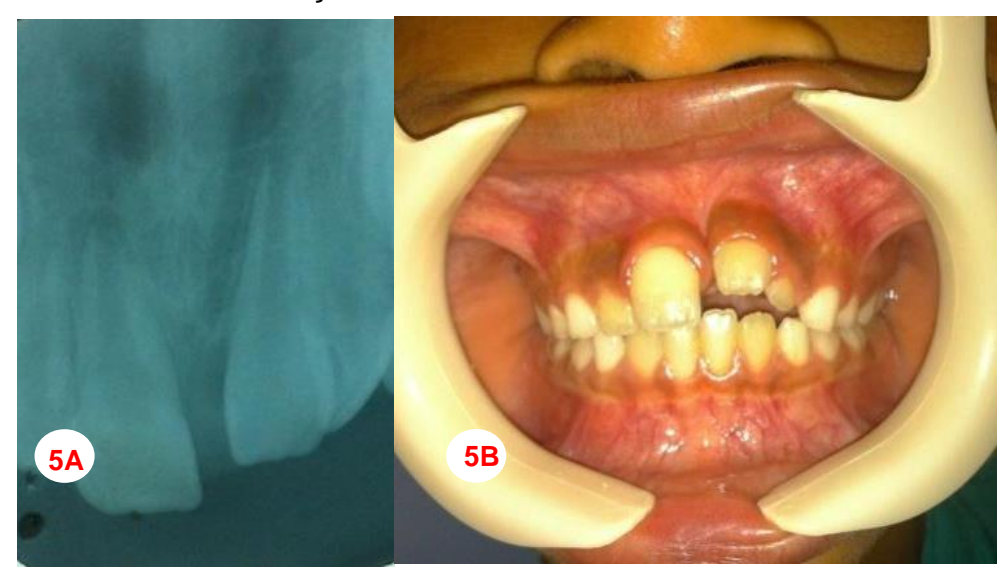

Fonte: Nogueira JSE, et al., 2020. 
Figura 6A/6B - Avaliação clínica e radiográfica após 12 meses sem complicações pósoperatórias.

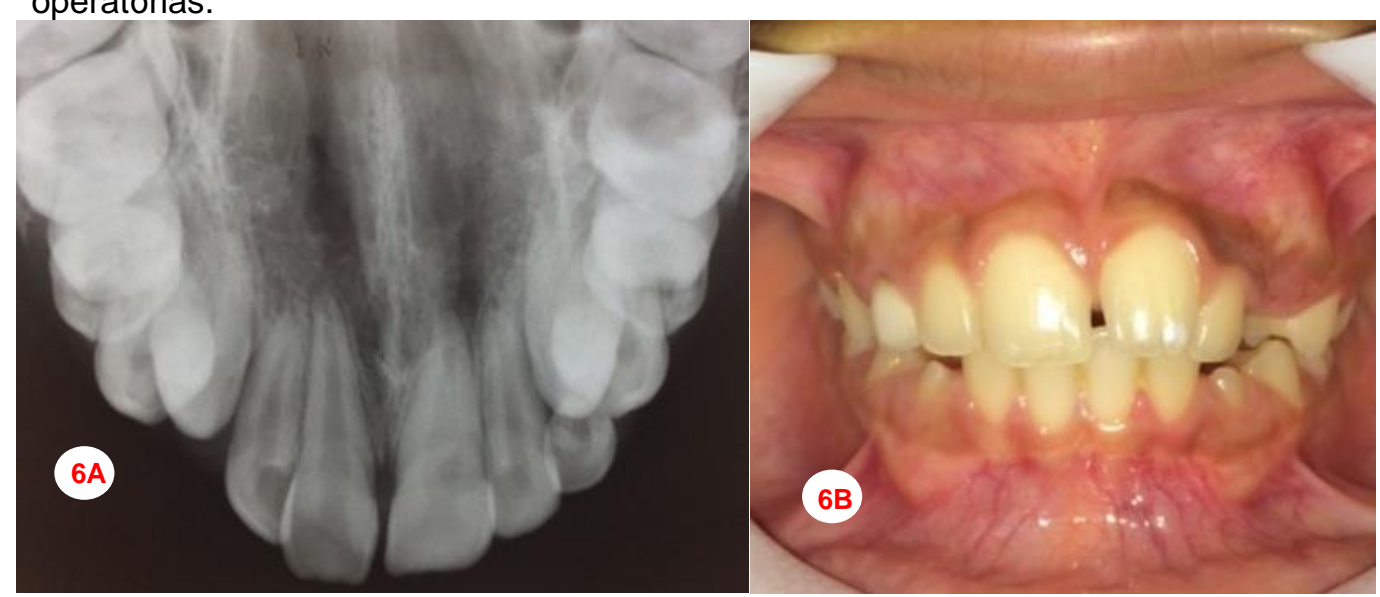

Fonte: Nogueira JSE, et al., 2020.

\section{DISCUSSÃO}

Apesar de bem documentado na literatura estudar e relatar casos de DSN é relevante para começarmos a compreender melhor sua etiologia e até chegar a métodos de prevenção a fim de evitar futuramente possíveis procedimentos invasivos (AI DHAFEERI HO, et al., 2014). O interessante e diferencial no caso acima relatado é que os DSN chegou ao serviço de urgência com a queixa de um "dente em formato anormal" sendo que ao exame clínico intraoral pode-se observar e constar que o mesiodens estava erupcionado logo seu diagnóstico não foi em um exame de rotina como é relatado comumente na literatura (DHULL KS, et al., 2012).

Embora o diagnóstico sugestivo imediato pelos cirurgiões responsáveis tenha sido devido aos sinais e sintomas clínicos apresentados como tumefação na região anterior de maxila fez-se necessário descartar a hipótese de patologias como odontomas ou demais tumores de origem odontogênica (SYRIAC G, et al., 2017; KUMAR DK e GOPAL KS, 2013). De acordo com a literatura a maioria dos casos, o diagnóstico é feito através de exames radiográficos de rotina onde cerca de $75 \%$ dos pacientes afetados apresentam DSN impactados e assintomáticos, logo estabelecer um protocolo pediátrico de acompanhamento e tomadas radiográficas semestrais ou anuais no início da dentição mista podem ajudar precocemente a diagnosticar casos de DSN (NANDINI DB, et al., 2014; LARA TS, et al., 2013).

No caso supra relatado o paciente foi submetido as radiografias panorâmica, oclusal e periapicais para os fins de diagnóstico, tendo em vista o baixo custo, a facilidade de execução e o resultado imediato (BELLO S, et al., 2019). Ainda que na literatura dados clínicos apontem que em certos casos a visualização de DSN na região anterior da maxila seja complicada devido à sobreposição de imagens, além de fatores como o processamento radiográfico que pode influenciar na imagem (GOKSEL S, et al., 2018).

Ademais, atualmente a tomografia computadorizada de feixe cônico (TCFC) é um recurso de diagnóstico bem documentado na literatura como método de diagnóstico por imagem superior aos exames radiográficos convencionais principalmente pela capacidade de análise tridimensional, melhor reprodução das estruturas anatômicas e menor distorção e artefatos de imagem (ZIEGLER CM e KLIMOWICZ TR, 2013). Contudo devemos sempre considerar as condições socioeconômicas e como supracitado paciente era de origem humilde logo tornou-se inviável neste caso o uso de TCFC (MOHAN S, et al., 2012).

Os DSN são uma forma de distúrbio de desenvolvimento associado ao aumento do número de dentes podendo receber também a denominação de hiperdontia, que pode representar cerca de 1 a $3 \%$ de todas as anomalias dentárias. Segundo Bello S, et al. (2019) a prevalência pode sofrer variações devidos a fatores demográficos, em especial, sobre idade, gênero e raça. Ainda segundo o autor a idade é fator de influência pois DSN são geralmente assintomáticos, como corrobora este relato de caso, sendo a segunda e a terceira décadas de vida os períodos mais incidentes para o diagnóstico de DSN, logo esses dados apontam falhas 
na adesão dos pais ao tratamento pediátrico e no sistema de avaliação e acompanhamento desses pacientes na primeira década de vidas (BELLO S, et al., 2019).

Sobre a influência do gênero na prevalência ainda não há nenhuma comprovação clínica, porém, a literatura cita o fator hormonal como fato de risco aos DSN. Ao analisarmos a prevalência por raça verificamos que DSN populações leucodermas possuem menor prevalência se comparados a populações melanodermas. Ainda sobre prevalência e incidência verificamos uma predileção pela maxila sobre a mandíbula na proporção de 5:1 ou 10:1 e tal predileção pode ocorrer pelo tecido ósseo trabeculado, pneumático e com maior vascularização da maxila, outro fator de influência pode ser que durante o desenvolvimento craniofacial intrauterino entre a $4^{\mathrm{a}}$ e $5^{\mathrm{a}}$ semanas, quando ocorre a formação do arco faríngeo que originará maxila e mandíbula, bem como a odontogênese que consiste num complexo processo de desenvolvimento dentário devido as interações entre o epitélio oral com o ectomesênquima.

Pode ser que essas interações tenham predisposição ao hiperdesenvolvimento da odontogênese pela maxila devido a facilidade de mobilidade do processo facial, durante o desenvolvimento craniofacial que pode ocasionar a ruptura da lâmina dentária acarretando na proliferação exagerada aumento as chances de DSN na maxila (BELLO S, et al., 2019; GOKSEL S, et al., 2018; CORTÉS-BRETÓN-BRINKMANN J, et al., 2019; ALSANI A e BALHADDAD AA, 2018).

Além disso há teorias que associação os DSN com traumas mecânicos que causariam uma hiperatividade da lâmina dentária na primeira década de vida e sendo a maxila uma região mais proeminente do que a mandíbula isso poderia explicar a maior incidência dos DSN na maxila principalmente na região anterior. Contudo a teoria mais aceita e difundida na odontopediatria é a Teoria da Hiperatividade da Lâmina Dentária cujo a produção de germes dentários seria acelerada ocasionando DSN. Outra teoria para justificar a maior incidência dos DSN na maxila seria sua presença em pacientes com fissuras labiais ou palatinas que naturalmente ocorrem na maxila (GUPTA A, et al., 2015).

Ao pesquisar na literatura verificamos que abordagens de tratamentos são diversas sendo que abordagens multidisciplinares geralmente. Estabelece que dentre as opções de tratamento para DSN não há um protocolo padrão estabelecidos na pediatria e/ou ortodontia sendo indicado a individualização do tratamento a partir do seu diagnóstico. A de exemplo o caso relatado neste artigo, a qual o mesiodens erupcionou na linha média e além de impactar a erupção do 21 e 22 ainda causava desconforto na língua ao paciente durante fala e mastigação, a abordagem não pode ser similar em casos de DSN incluso na região posterior de maxila (CORTÉS-BRETÓN-BRINKMANN J, et al., 2019; ALSANI A e BALHADDAD AA, 2018).

\section{CONSIDERAÇÕES FINAIS}

Com este estudo de caso conclui-se que consultas preventivas na odontopediatria são importantes, pois através de exames periódicos é possível detectar anomalias e patologias. Exames clínicos e radiográficos são fundamentais para se obter um bom diagnóstico precoce e planejamento cirúrgico adequado. O cirurgião dentista deve sempre dá importância a intervenção preventiva com o objetivo de evitar danos futuros à dentição permanente do paciente pediátrico.

\section{REFERÊNCIAS}

1. DAS D, MISRA J. Surgical management of impacted incisors in associate with supernumerary teeth: a combine case report of spontaneous eruption and orthodontic extrusion. J Indian Soc Pedod Prev Dent. 2012; 30(4): 329-32.

2. RALLAN M, et al. Surgical management of multiple supernumerary teeth and an impacted maxillary permanent central incisor. BMJ Case Rep. 2013; bcr2013009995.

3. WAINGADE M, et al. Pindborg tumor arising in association with an impacted supernumerary tooth in the anterior maxilla. J Mich Dent Assoc. 2014; 96(6): 26-9.

4. BARGALE SD, et al. Bilateral supernumerary primary teeth in the maxillary anterior region. BMJ Case Rep. 2015; 24; bcr2015210663.

5. COSME-SILVA L, et al. Combined Surgical Removal of a Supernumerary Tooth and Orthodontic Traction of an Impacted Maxillary Central Incisor. J Dent Child (Chic). 2016; 15; 83(3): 167-172. 
6. SARNE O, et al. Supernumerary Teeth in the Maxillary Anterior Region: The Dilemma of Early Versus Late Surgicallntervention. J Clin Pediatr Dent. 2018; 42(1): 55-61.

7. GURLER G, DELILBASI C, DELILBASI E. Investigation of impacted supernumerary teeth: a cone beam computed tomograph (cbct) study. J Istanb Univ Fac Dent. 2017; 2; 51(3): 18-24.

8. INDIRA M, et al. Molariform mesiodens in primary dentition: a case report. J Clin Diagn Res. 2014 May; 8(5): ZD335.

9. MANGALEKAR SB, et al. Molariform mesiodens in primary dentition. Case Rep Dent. 2013; 2013: 750107.

10. THOMAIDIS V, TSOUCALAS G, FISKA A. Rotated mesiodens in children. An immediate surgical removal or active monitoring? Clin Case Rep. 2019; 17; 7(12): 2577-2578.

11. MADDALONE M, et al. Evaluation of Surgical Options for Supernumerary Teeth in the Anterior Maxilla. Int $J$ Clin Pediatr Dent. 2018; 11(4): 294-298.

12. HAUER L, et al. Modified maxillary vestibular approach with subperiostal intranasal dissection for surgical extractions of mesiodentes impacted in the floor of the nasal cavity. J Craniomaxillofac Surg. 2019; 47(1): 1-5.

13. OZDEN MC, et al. Bilateral molariform supernumerary teeth in the anterior maxilla: a report of two cases. $\mathrm{J}$ Istanb Univ Fac Dent. 2017; 2, 51(1): 57-60.

14. AL DHAFEERI HO, et al. Recurrent epistaxis caused by an intranasal supernumerary tooth in a young adult. Am J Case Rep. 2014; 5, 15: 291-3.

15. DHULL KS, et al. Bilateral maxillary paramolars: a case report. J Dent Child (Chic). 2012; 79(2): 84-7.

16. SYRIAC G, et al. Prevalence, Characteristics, and Complications of Supernumerary Teeth in Nonsyndromic Pediatric Population of South India: A Clinical and Radiographic Study. J Pharm Bioallied Sci. 2017; (Suppl 1): S231-S236.

17. KUMAR DK, GOPAL KS. An epidemiological study on supernumerary teeth: a survey on 5,000 people. J Clin Diagn Res. 2013; 7(7): 1504-7.

18. NANDINI DB, et al. Diagnostic dilemma of a double tooth: a rare case report and review. J Clin Diagn Res. 2014; 8(1): 271-2.

19. LARA TS, et al. Prevalence of mesiodens in orthodontic patients with deciduous and mixed dentition and its association with other dental anomalies. Dental Press J Orthod. 2013; 18(6): 93-9.

20. ZIEGLER CM, KLIMOWICZ TR. A comparison between various radiological techniques in the localization and analysis of impacted and supernumerary teeth. Indian J Dent Res. 2013; 24(3): 336-41.

21. MOHAN S, KANKARIYA H, FAUZDAR S. Impacted Inverted Teeth with their Possible Treatment Protocols. J Maxillofac Oral Surg. 2012; 11(4): 455-7.

22. BELLO S, et al. Prevalence and presentation of hyperdontia in a non-syndromic, mixed Nigerian population. J Clin Exp Dent. 2019; 11(10): e930-e936.

23. GOKSEL S, et al. Evaluation of Prevalence and Positions of Mesiodens Using Cone-Beam Computed Tomography. J Oral Maxillofac Res. 2018; 30, 9(4): e1.

24. CORTÉS-BRETÓN-BRINKMANN J, et al. Clinical repercussions and epidemiological considerations of supernumerary canines: A 26 case series. Med Oral Patol Oral Cir Bucal. 2019; 1, 24(5): e615-e620.

25. ALSANI A, BALHADDAD AA. Delayed Eruption of Maxillary Central Incisors Associated with the Presence of Supernumerary Teeth: A Case Report with 18 Months Follow-up. J Contemp Dent Pract. 2018; 19(12): 1434-1436.

26. GUPTA A, et al. Hyperactive Dental Lamina in a 24-Year-old Female - A Case Report and Review of Literature. J Clin Diagn Res. 2015; 9(8): ZE01-4. 